\title{
Comparison of psychoacoustic-based reverberance parameters
}

\author{
Doheon Lee, ${ }^{1, a)}$ Jasper van Dorp Schuitman, ${ }^{2}$ Densil Cabrera, ${ }^{3}$ Xiaojun Qiu, ${ }^{1}$ \\ and Ian Burnett ${ }^{1}$ \\ ${ }^{1}$ Faculty of Engineering and Information Technology, University of Technology, Sydney, New South Wales \\ 2007, Australia \\ ${ }^{2}$ Sound Intelligence, Amersfoort, The Netherlands \\ ${ }^{3}$ Sydney School of Architecture, Design and Planning, The University of Sydney, Sydney, New South Wales \\ 2006, Australia
}

(Received 28 March 2017; revised 6 September 2017; accepted 14 September 2017; published online 5 October 2017)

\begin{abstract}
This study compared psychoacoustic reverberance parameters to each other, as well as to reverberation time (RT) and early decay time (EDT) under various acoustic conditions. The psychoacoustic parameters were loudness-based RT $\left(T_{N}\right)$, loudness-based EDT [EDT $N$; Lee, Cabrera, and Martens, J. Acoust. Soc. Am. 131, 1194-1205 (2012a)], and parameter for reverberance $\left[P_{\text {REV }}\right.$; van Dorp Schuitman, de Vries, and Lindau., J. Acoust. Soc. Am. 133, 1572-1585 (2013)]. For the comparisons, a wide range of sound pressure levels (SPLs) from $20 \mathrm{~dB}$ to $100 \mathrm{~dB}$ and RTs from $0.5 \mathrm{~s}$ to $5.0 \mathrm{~s}$ were evaluated, and two sets of subjective data from the previous studies were used for the cross-validation and comparison. Results of the comparisons show that the psychoacoustic reverberance parameters provided better matches to reverberance than RT and EDT; however, the performance of these psychoacoustic reverberance parameters varied with the SPL range, the type of audio sample, and the reverberation conditions. This study reveals that $P_{\mathrm{REV}}$ is the most relevant for estimating a relative change in reverberance between samples when the SPL range is small, while $\mathrm{EDT}_{N}$ is useful in estimating the absolute reverberance. This study also suggests the use of $P_{\mathrm{REV}}$ and $\mathrm{EDT}_{N}$ for speech and music samples, respectively.

(C) 2017 Acoustical Society of America. https://doi.org/10.1121/1.5005508
\end{abstract}

Pages: $1832-1840$

\section{INTRODUCTION}

Reverberation is one of the most important physical phenomena in room acoustics. To estimate reverberation, ISO 3382-1 (2009) recommends reverberation time (RT) and early decay time (EDT; hereafter, referred to as ISO reverberation parameters); however, these parameters do not represent the human perception of reverberation (hereafter, referred to as reverberance) well in some acoustic scenarios (e.g., Barron, 1988; Lokki et al., 2012). This is, in part, due to auditory perception being a complex psychoacoustic process while the sound pressure level (SPL) decay values from which RT and EDT are derived represent very little of the transformation from sound to perception (Fastl and Zwicker, 2007).

To better estimate reverberance, Lee et al. (2012a) and van Dorp Schuitman et al. (2013) independently proposed psychoacoustic reverberance parameters. Although these parameters were shown to provide closer matches to reverberance than RT and EDT, they do not necessarily perform equally well because the parameters are derived from the output of different psychoacoustic models and emphasize different attributes of reverberance. In the present study, these psychoacoustic reverberance parameters are compared under various acoustic conditions to determine those that best represent reverberance over a range of acoustic conditions, and to better understand the strengths and limitations of these approaches.

a)Electronic mail: dosyd@hotmail.com
The major differences between the psychoacoustic reverberance parameters are as follows: (1) Loudness-based RT $\left(T_{N}\right)$ and loudness-based EDT $\left(\mathrm{EDT}_{N}\right)$ by Lee et al. (2012a) are based on the analysis of a room impulse response (RIR) in conjunction with the SPL, while parameter for reverberance $\left(P_{\mathrm{REV}}\right)$ by van Dorp Schuitman et al. $(2013)$ is calculated directly from a running signal, e.g., music and/or speech; (2) $T_{N}$ and $\mathrm{EDT}_{N}$ are based on the notion that reverberance comes from the perception of sound decay, while

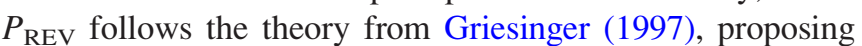
that reverberance is related to the absolute level of the room's reverberation and is independent of the level of the direct sound; and (3) the time-varying loudness model (TVL; Glasberg and Moore, 2002) and dynamic loudness model (Chalupper and Fastl, 2002) were used for $T_{N}$ and $\mathrm{EDT}_{N}$, while an auditory model based on the work by Breebaart (2001) and Breebaart et al. (2001) was used for $P_{\text {REV }}$.

On the basis of (2), the two sets of parameters are calculated in different ways. The calculation of $T_{N}$ and $\mathrm{EDT}_{N}$ is similar to RT and EDT, i.e., a RIR is processed with a loudness model, and the decay time of its loudness decay function is calculated by analogy to RT and EDT. The slope of a loudness decay function becomes less steep as the SPL of a RIR increases (see Fig. 1 in Lee et al., 2012a), so $T_{N}$ and $\mathrm{EDT}_{N}$ values of a given RIR depend on the assumed listening level. For $P_{\mathrm{REV}}$, a running signal is processed with the auditory model based on the work by Breebaart (Breebaart, 2001; Breebaart et al., 2001), and the monaural outputs of the peripheral processor in the auditory model (which 
models outer/middle ear transfer function, basilar membrane, hair cells, and neural firing) are split into a foreground and background stream (as suggested by Griesinger, 1997; Rumsey, 2002; Mason et al., 2004). Then, $P_{\mathrm{REV}}$ is calculated as the average level of the background stream.

The differences in calculation method lead to distinct advantages and disadvantages of the parameters. As $P_{\mathrm{REV}}$ is based on a running signal, this parameter can account for the influence of sample on reverberance (Osses Vecchi et al., 2017), which can be an important contributor to reverberance (Teret et al., 2017). Furthermore, $P_{\mathrm{REV}}$ can estimate different characteristics of reverberance (such as stopped reverberance, running reverberance, and overall reverberance) (Morimoto and Asaoka, 2004) on the basis of analysing a selected part of a sample, and can be calculated both from audio recordings and during a live concert situation. However, for the same reason, a particular source-receiver pair of positions in a room can have different values of $P_{\mathrm{REV}}$ depending on which sample is selected and the processing thereof (e.g., inclusion or exclusion of silence at the end of the sample). In contrast, $T_{N}$ and $\mathrm{EDT}_{N}$ are based on a RIR in conjunction with SPL, and beyond these the values are not influenced by the particular features of a running signal. Hence, these parameters are insensitive to some features of a sample that may affect reverberance. Furthermore, they cannot be calculated from live concert signals nor from audio recordings.

For the comparisons in this study, the parameters are calculated over a wide range of SPLs and RTs, and the cross-validation is performed with two sets of subjective data collected in the previous studies of the authors, i.e., Lee et al. (2012a) and van Dorp Schuitman et al. (2013). For the $T_{N}$ and $\mathrm{EDT}_{N}$ calculations, the short-term loudness output of the TVL is used, since it approximates the momentary loudness perception of a binaural input signal (Moore and Glasberg, 2007). For the $P_{\mathrm{REV}}$ calculations, the auditory model based on the work by Breebaart (Breebaart, 2001; Breebaart et al., 2001) is used and the final decay of the music and speech is excluded from the analysis as in the study by van Dorp Schuitman (2011).

In Secs. II and III, the effect of SPL and $T_{20}$ (i.e., RT with its evaluation range from $-5 \mathrm{~dB}$ to $-25 \mathrm{~dB}$ ) on $T_{N}, \mathrm{EDT}_{N}$, and $P_{\mathrm{REV}}$ are investigated, and then two sets of listening experiments performed by Lee et al. (2012a) and van Dorp Schuitman et al. (2013) are briefly described and the performance of the psychoacoustic reverberance parameters assessed. Finally, the main results are discussed and conclusions drawn.

\section{EFFECT OF SPL AND $T_{20}$ ON PSYCHOACOUSTIC PARAMETERS}

In this section, the psychoacoustic reverberance parameters are calculated for various SPL and $T_{20}$ values. $T_{N}$ and $\mathrm{EDT}_{N}$ values are calculated from RIRs measured in a medium sized concert auditorium (Farina and Ayalon, 2003), and in a lecture theatre at the University of Sydney. The auditorium has 1200 seats with $T_{20}$ of $1.84 \mathrm{~s}$; the lecture theatre has 162 seats with $T_{20}$ of $0.92 \mathrm{~s}$. For $P_{\mathrm{REV}}$ calculations, these RIRs are convolved with an anechoic music excerpt from the
Denon Professional Test CD No. 2 (1988), i.e., Overture to The Marriage of Figaro by Mozart.

Figure 1 shows the effect of SPL on the psychoacoustic reverberance parameters, where $L_{\text {Aeq }}$ (which is the poweraveraged A-weighted SPL over a given time period) of the music sample ranges from $20 \mathrm{~dB}$ to $100 \mathrm{~dB}$ at $5 \mathrm{~dB}$ intervals. For $T_{N}$ and $\mathrm{EDT}_{N}$ calculations, $L_{\mathrm{AFmax}}$ (which is the maximum A-weighted SPL with a "fast" temporal integration, i.e., using a $125 \mathrm{~ms}$ constant) of the RIRs is adjusted to these $L_{\text {Aeq }}$ values. This range includes the SPLs for which $T_{N}, \mathrm{EDT}_{N}$, and $P_{\mathrm{REV}}$ were validated in previous studies, e.g., from $60 \mathrm{~dB}$ to $80 \mathrm{~dB}$ in $L_{\text {Aeq }}$ for $T_{N}$ and $\mathrm{EDT}_{N}$ (see Table I, in Lee et al., $2012 \mathrm{~b}$ ), and from $47 \mathrm{~dB}$ to $79 \mathrm{~dB}$ in $L_{\mathrm{eq}}$ (which corresponds to approximately $43 \mathrm{~dB}-72 \mathrm{~dB}$ in $L_{\mathrm{Aeq}}$ ) for $P_{\mathrm{REV}}$ (see Table I in van Dorp Schuitman et al., 2013). It should be noted that in the previous studies the parameters were not calculated at $5 \mathrm{~dB}$ intervals, and therefore the SPLs tested in Fig. 1 are not exactly the same as those tested in the previous studies.

As shown in Fig. 1, the psychoacoustic reverberance parameters increase with the SPL, which reflects a positive relationship between reverberance and listening level (Lee and Cabrera, 2010; Hase et al., 2000). Figure 1 shows that $P_{\mathrm{REV}}$ values rise more steeply with SPLs from $60 \mathrm{~dB}$ to $100 \mathrm{~dB}$ than at lower SPLs. Compared to $P_{\mathrm{REV}}, T_{N}$, and $\mathrm{EDT}_{N}$ values increase more gradually over the entire SPL range, and they stop increasing or start decreasing at around $90 \mathrm{~dB}$.

Figure 2 shows the variations of the psychoacoustic reverberance parameters for $T_{20}$ ranging from $0.5 \mathrm{~s}$ to $5.0 \mathrm{~s}$

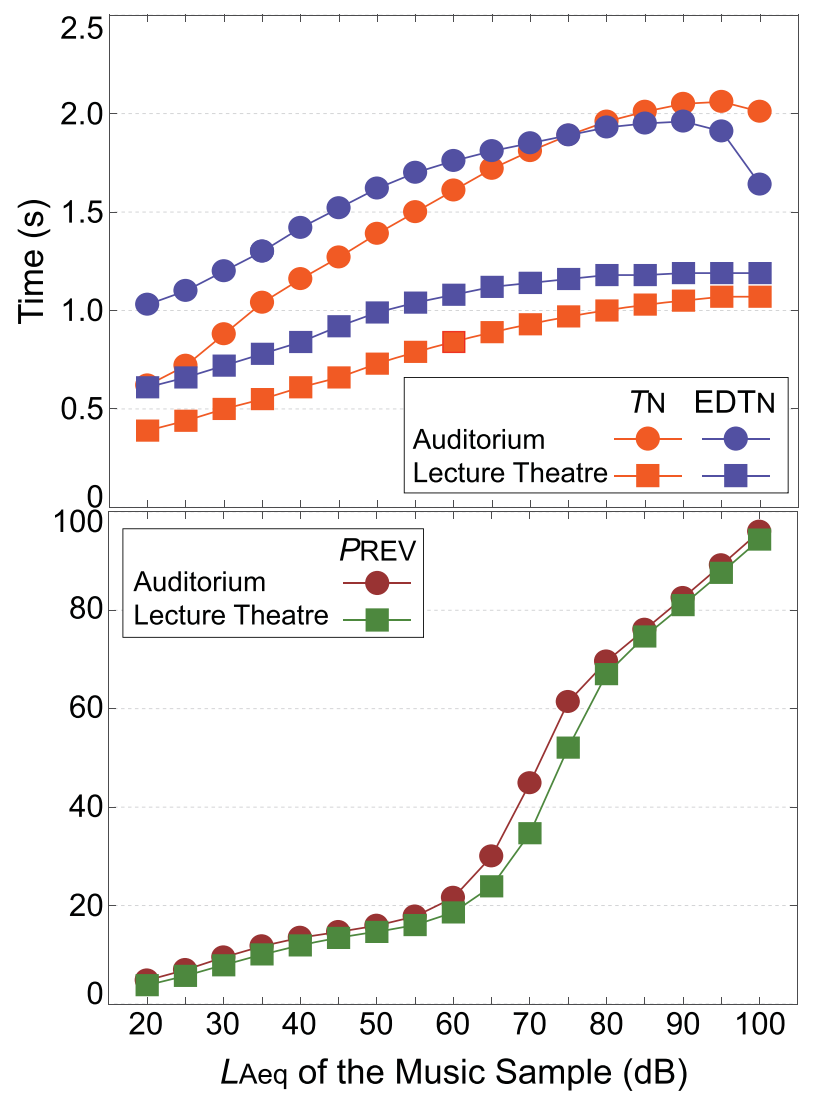

FIG. 1. (Color online) $T_{N}, \mathrm{EDT}_{N}$, and $P_{\mathrm{REV}}$ as functions of the SPL from $20 \mathrm{~dB}$ to $100 \mathrm{~dB}$ with $T_{20}$ being 1.84 (Auditorium) and $0.92 \mathrm{~s}$ (Lecture Theatre). The unit of $T_{N}$ and $\mathrm{EDT}_{N}$ is time in seconds, and $P_{\mathrm{REV}}$ is a unitless parameter. 
TABLE I. An overview of the properties of experiments A.

\begin{tabular}{lcccccccc}
\hline \hline & S1 & S2 & M1 & M2 & M3 & L1 & L2 & L3 \\
\hline Source-receiver distance $(m)$ & 12 & 24 & 10 & 19 & 31 & 20.5 & 30 & 48 \\
EDT $_{\text {mid }}(\mathrm{s})$ & 1.89 & 1.98 & 1.83 & 1.77 & 2.00 & 2.44 & 2.25 & 2.38 \\
$T_{20, \text { mid }}(\mathrm{s})$ & 2.06 & 2.07 & 2.01 & 2.03 & 2.17 & 2.66 & 2.60 & 2.53 \\
Experiment A.1, $L_{\text {Aeq }}(\mathrm{dB})$ & 76.0 & 75.6 & 75.5 & 73.7 & 72.4 & 71.3 & 70.7 & 65.1 \\
Experiment A.2, $L_{\text {Aeq }}(\mathrm{dB})$ & 77.5 & 76.1 & 76.8 & 74.6 & 73.5 & 72.1 & 71.1 & 65.2 \\
\hline \hline
\end{tabular}

with $0.5 \mathrm{~s}$ intervals. For this comparison, $T_{20}$ of the auditorium RIR used for Fig. 1 was changed by modifying its decay envelope in the method suggested by Cabrera et al. (2011). To summarise the method, the RIR was processed with octave-band filters from $32.5 \mathrm{~Hz}$ to $16 \mathrm{kHz}$, and noise floors that come after the reverberation decays of the filtered RIRs were decayed at the same rate of the corresponding reverberation decay envelope. Then, the noise-treated octave-band RIRs were multiplied by exponential functions

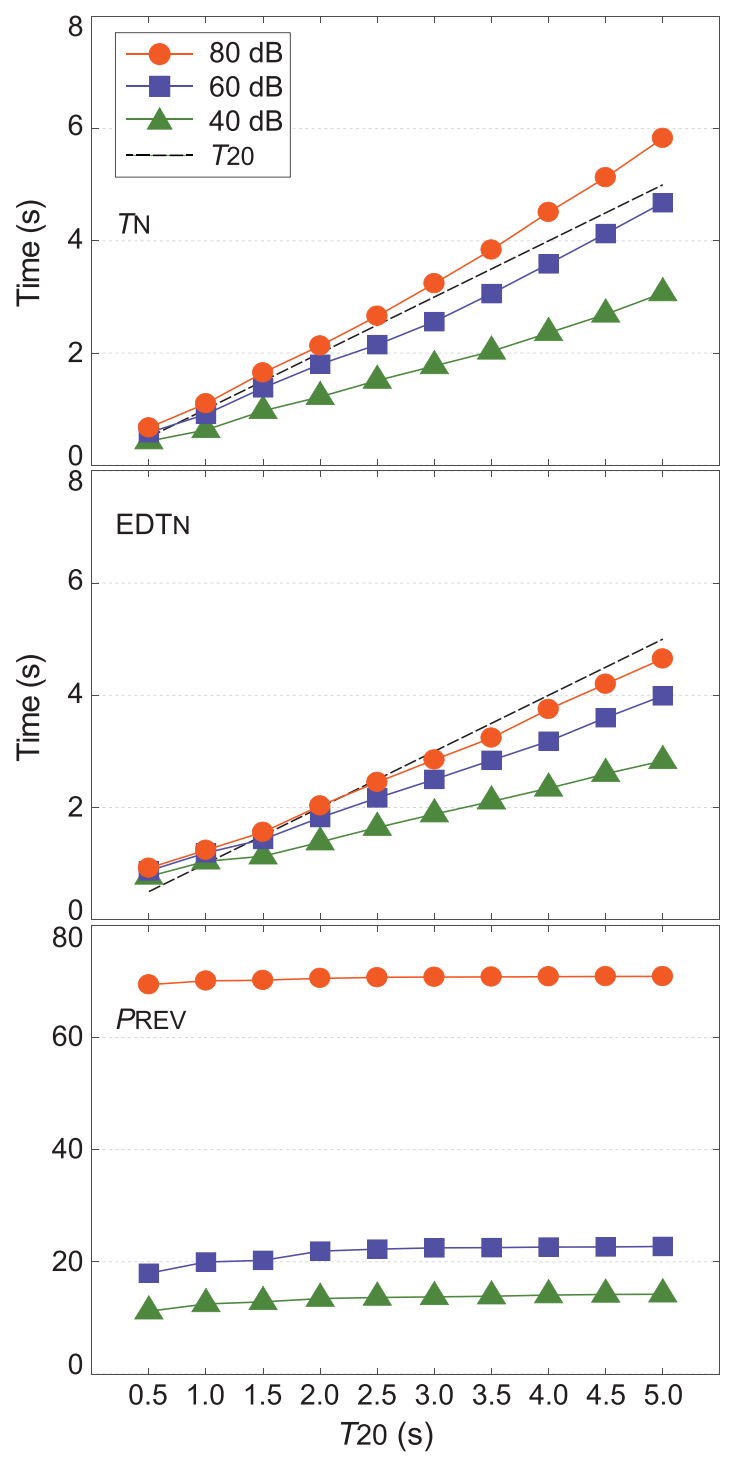

FIG. 2. (Color online) $T_{N}, \mathrm{EDT}_{N}$, and $P_{\mathrm{REV}}$ as functions of $T_{20}$ from $0.5 \mathrm{~s}$ to $5.0 \mathrm{~s}$, with the SPLs being $40 \mathrm{~dB}, 60 \mathrm{~dB}$, and $80 \mathrm{~dB}$. The unit of $T_{N}$ and $\mathrm{EDT}_{N}$ is time in seconds, and $P_{\mathrm{REV}}$ is a unit-less parameter. to achieve the desired octave-band RTs and combined into a single RIR.

In Fig. 2, $T_{N}$ and $\mathrm{EDT}_{N}$ values increase almost linearly with $T_{20}$, and the effect of $T_{20}$ becomes stronger as the SPL increases. For $T_{N}$ at $60 \mathrm{~dB}$ and $80 \mathrm{~dB}$ and $E_{N}$ at $80 \mathrm{~dB}$, these parameters increase at a rate similar to that of $T_{20}$. In contrast, $P_{\text {REV }}$ is scarcely affected by $T_{20}$, but is strongly affected by the SPL. For example, a tenfold increase in $T_{20}$ (from $0.5 \mathrm{~s}$ to $5.0 \mathrm{~s}$ ) increases $P_{\mathrm{REV}}$ at $80 \mathrm{~dB}$ by approximately $2 \%$ only (from 69.41 to 70.88 ). However, when the SPL increases from $40 \mathrm{~dB}$ to $80 \mathrm{~dB}, P_{\mathrm{REV}}$ at $T_{20}$ of $1.5 \mathrm{~s}$ is increased by more than five times (from 12.86 to 70.19 ).

It should be noted that, in previous studies, $T_{N}$ and $\mathrm{EDT}_{N}$ have been validated for $T_{20}$ from $1.0 \mathrm{~s}$ to $3.0 \mathrm{~s}$ (Lee et al., 2012b), and $P_{\mathrm{REV}}$ has been validated for $T_{20}$ from $0.02 \mathrm{~s}$ to $10.12 \mathrm{~s}$ (van Dorp Schuitman et al., 2013). However, the tested $T_{20}$ values in the previous studies were not at $0.5 \mathrm{~s}$ intervals. For this reason, $T_{20}$ values in Fig. 2 are not exactly the same as those tested in the previous studies, although they are in the same range.

\section{CROSS VALIDATION}

In this section, cross-validation is performed with the subjective data collected in the studies of Lee et al. (2012a) and van Dorp Schuitman et al. (2013) in order to discover the acoustic conditions in which each psychoacoustic reverberance parameter performs best (either by yielding the lowest coefficient of variation for reverberance-matched samples or the highest correlation coefficient with the collected subject responses).

\section{A. Matching reverberance experiments}

\section{Experiments}

Lee et al. (2012a) performed listening experiments with two types of samples: orchestral music and a tenor solo voice singing an operatic excerpt. Eight RIRs measured by Farina and Ayalon (2003) in three auditoria were convolved with the anechoic recordings to generate the base stimuli. The detailed acoustic conditions are given in Table I, and are labeled to indicate the auditorium size [i.e., small (S), medium (M), and large (L)] and a number ordinally indicating source-to-receiver distance. It should be noted that the values in Table I are from RIRs with the noise floor treatment (in the same way applied for Fig. 2), which was necessary to avoid auralization artefacts when RT was increased as part of the listening test [see Eq. (1)]. The signal-to-noise ratios of the original RIRs were higher than $60 \mathrm{~dB}$, and the noise floor treatment was applied from the point that is $10 \mathrm{~dB}$ above the noise floor. The treatment changed $\mathrm{EDT}_{\text {mid }}$ and $T_{20 \text {,mid }}$ of the RIRs by less than $0.01 \mathrm{~s}$ (hereafter, the subscript "mid" refers to an average of its value at $500 \mathrm{~Hz}$ and $1 \mathrm{kHz}$ in octave bands, and the subscript "oct" refers to an average of its values from $125 \mathrm{~Hz}$ to $4 \mathrm{kHz}$ in octave bands).

In this paper, the experiment with the instrumental music samples is referred to as experiment A.1 and the experiment with a tenor voice is referred to as experiment 
A.2. Note that Lee et al. (2012a) investigated the effect of SPL on reverberance by adding $\pm 5 \mathrm{~dB}$ gains to the convolution products, so the actual SPLs tested in that study were approximately from $60 \mathrm{~dB}$ to $82 \mathrm{~dB}$ in $L_{\mathrm{Aeq}}$.

The task was to match the reverberance of 24 comparison samples $(8$ RIRs $\times 3$ gains of $-5,0$, and $+5 \mathrm{~dB})$ to the reverberance of one reference sample. The reference sample in each experiment was the respective anechoic recording convolved with the RIR identified as M1 (Table I). The subjects listened to 24 pairs of samples that consisted of the reference sample and one of the 24 comparison samples, and pressed "more" or "less" buttons on a MATLAB (MathWorks, Natick, MA) graphical user interface to adjust the reverberation decay of RIRs, which was then convolved with the anechoic samples for listening. Pressing the buttons incremented or decremented $d$ in Eq. (1), where the $p(t)$ is sound pressure of a RIR as a function of time, $t$ is time in seconds, and $p^{\prime}(t)$ is sound pressure of the RIR as a function of time after the reverberant decay has been adjusted.

A unit step of $d$ corresponds to approximately a $4 \%$ change in $\mathrm{EDT}_{\text {mid }}$, which is similar to the $5 \%$ just noticeable difference (JND) of reverberance specified in ISO 3382-1 (2009). The initial value of $d$ for the comparison samples was randomly chosen from -7 to 7 , so that the samples were played with randomized reverberance at first. It should be noted that Eq. (1) changes the energy of a RIR, and this is compensated by multiplying $\Delta L_{E}$ in Eq. (2) to RIRs before the convolution process. That way, an undesired change in $L_{\text {Aeq }}$ due to the change in reverberation decay of a RIR is avoided:

$$
\begin{aligned}
& p^{\prime}(t)=p(t) \exp \left(\frac{\left(-3+\left(3 \times 1.04^{d}\right) t\right)}{1.04^{d}}\right), \\
& \Delta L_{E}=10 \log _{10}\left(\frac{\int p^{\prime 2}(t)}{\int p^{2}(t)}\right) .
\end{aligned}
$$

The samples were presented via circumaural headphones (Sennheiser HD600, Sennheiser, Hanover) in an anechoic chamber at the University of Sydney in Australia for experiment A.1 and in a listening booth at Tohoku University in Japan for experiment A.2. Twenty subjects and 15 subjects took part on a volunteer basis in experiments A.1 and A.2, respectively. None of the subjects self-reported any hearing loss. As one of the comparison samples was physically identical to the reference sample, subjects who mismatched the reverberance of this pair by more than two times the JND of the reverberance (i.e., a $10 \%$ change in $\mathrm{EDT}_{\text {mid }}$ ) were considered insensitive to the task and were excluded from analyses. To assess this, $\mathrm{EDT}_{\text {mid }}$ of the reference RIR is compared with that of the comparison RIR (which is physically identical to the reference RIR) incorporating the $d$-adjustment by each subject. The number of subjects excluded from the analyses was 4 in each experiment (note that they are not the same subjects), and therefore the responses from 16 and 11 subjects were analysed further for experiments A.1 and A.2, respectively.

\section{Results}

The subject responses represented by $d$ in Eq. (1) are given in Fig. 3. In Fig. 3, each box represents 25th, 50th, and 75th percentiles with whiskers extending to the most extreme $d$ values that are not outliers. Outliers marked with a red cross are $d$ values beyond 1.5 times the interquartile range from 25 th and 75 th percentiles. The interquartile range is defined as the difference between 25th percentile and 75th percentile. As shown in Fig. 3, M1 has median (i.e., 50th percentile) values close to zero (i.e., -0.5 and 0 in experiments A.1 and A.2, respectively), indicating that the subject matched reverberance correctly for the two physically identical samples. According to a three-way analysis of variance (ANOVA), the subject responses in $d$ are significantly affected by the additional SPL changes of $\pm 5 \mathrm{~dB}[F(2,614)$ $=33.81, p<0.01]$, the RIRs $[F(7,614)=10.66, p<0.01]$, and the experiment number $[F(1,614)=7.72, p<0.01]$. However, there is no significant interaction effect between these independent variables, i.e., $[F(7,614)=1.02$, $p=0.414]$ between the RIR and the experiment number; $[F(2,614)=0.15, p=0.857]$ between the gain and the experiment number; and $[F(14,614)=0.7, p=0.773]$ between the RIR and the gain.

The $T_{N}, \mathrm{EDT}_{N}$, and the ISO reverberation parameters were derived from the reverberance-matched RIRs (of which reverberation decays were adjusted using the averaged $d$ values). For $P_{\mathrm{REV}}$ calculations, these RIRs were convolved with the anechoic recordings of the orchestral music and the tenor voice singing an operatic excerpt.

The coefficient of variation between the 24 reverberanceadjusted samples was calculated for each parameter, and results are shown in Fig. 4. The coefficient of variation is the standard deviation divided by mean and, hence, a meanrelated bias in the standard deviation is eliminated. As reverberance of the 24 comparison samples was subjectively matched to the same reference reverberance, the coefficient of variation between the reverberance-adjusted samples is ideally

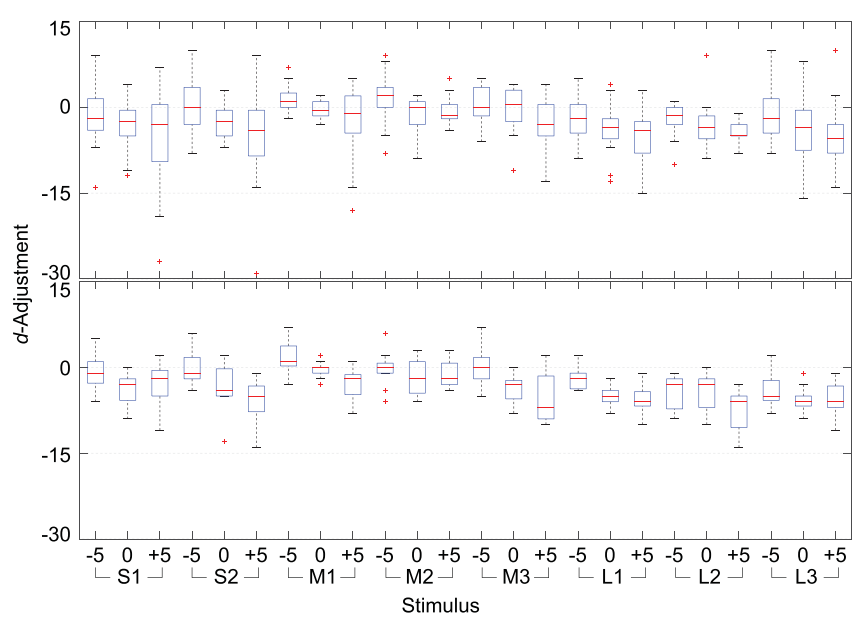

FIG. 3. (Color online) $d$-adjustments made by the subjects for matching reverberance [see Eq. (1)]. The numbers in the $x$ axis represent the additional SPL changes of $\pm 5 \mathrm{~dB}$ applied to the samples. Boxes represent $25 \mathrm{th}$, 50th (red line), and 75 th percentiles with whiskers extending to 1.5 times the interquartile range from 25 th and 75 th percentiles. Red crosses are outliers that are $d$ values beyond the whiskers. 


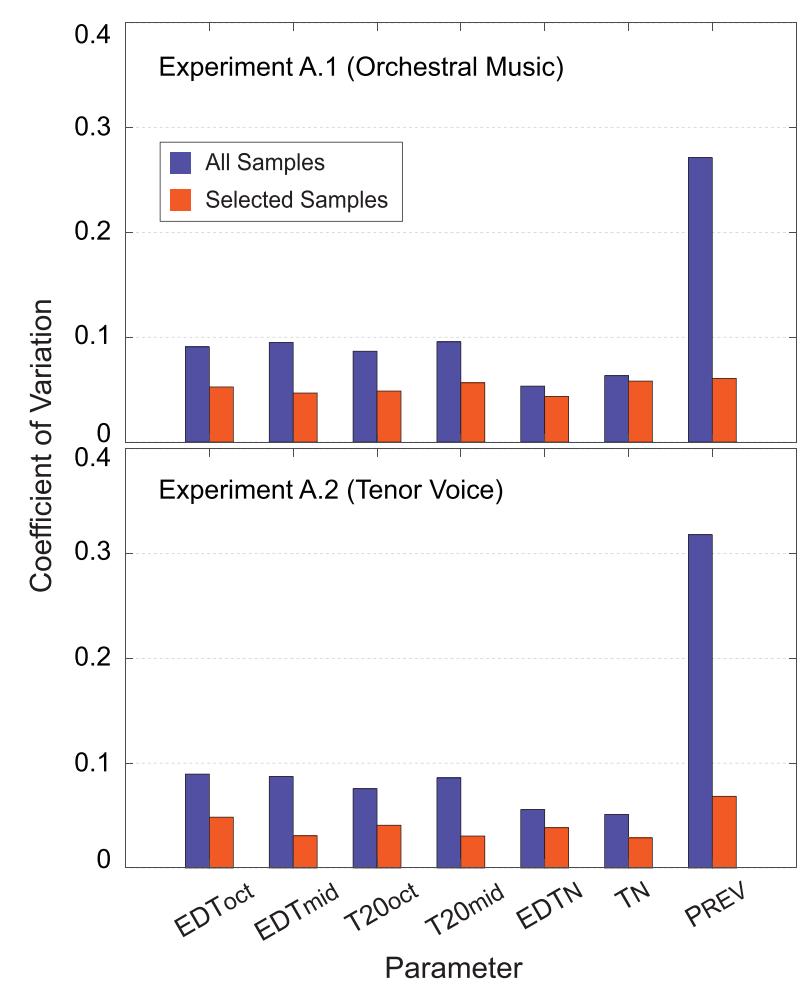

FIG. 4. (Color online) Values of the coefficient of variation for each parameter calculated from 24 reverberance-adjusted samples. The upper figure is for experiment A.1 (the orchestral music samples) and the lower figure is for experiment A.2 (the tenor voice singing an opera excerpt). The selected samples are S1 $(-5 \mathrm{~dB}), \mathrm{S} 2(-5 \mathrm{~dB}), \mathrm{M} 1(-5 \mathrm{~dB}), \mathrm{L} 1(0 \mathrm{~dB}), \mathrm{L} 2(0 \mathrm{~dB})$, and L3 $(+5 \mathrm{~dB})$, over which SPL range is less than $1 \mathrm{~dB}$. The coefficient of variation of an ideal parameter would be zero for the reverberance-adjusted samples.

zero. One set of results is obtained from analysing all the samples, and the other set is obtained from analysing only six RIRs: S1 $(-5 \mathrm{~dB}), \mathrm{S} 2(-5 \mathrm{~dB}), \mathrm{M} 1(-5 \mathrm{~dB}), \mathrm{L} 1(0 \mathrm{~dB}), \mathrm{L} 2$ $(0 \mathrm{~dB})$, and L3 $(+5 \mathrm{~dB})$, over which SPL difference is less than $1 \mathrm{~dB}$. Hence, the effect of SPL is eliminated in the analysis of the latter set.

For the analysis of all samples, $T_{N}$ and $\mathrm{EDT}_{N}$ have the lowest coefficients of variation, and $P_{\text {REV }}$ yields the highest coefficient of variation. The significance of differences between coefficients of variation is examined with a twosample $f$-test. It should be noted that this test is originally for testing the significance of difference between variances or between standard deviations. However, in this study, a twosample $f$-test was executed on values of each parameter divided by its mean (which is also necessary because the $P_{\mathrm{REV}}$ values are different in scale to the other values), so that the result shows the significance of difference between coefficients of variation because the coefficient of variation is the standard deviation divided by mean.

According to the result of a two-sample $f$-test, in experiment A.1 the coefficient of variation of $\mathrm{EDT}_{N}$ is significantly lower than the other parameters $(p<0.01$ for all pairs of parameters with $\mathrm{EDT}_{N}$ ) except between $\mathrm{EDT}_{N}$ and $T_{N}$ $[F(1,23)=0.71, p=0.425]$. In experiment A.2, the coefficients of variation of $\mathrm{EDT}_{N}$ and $T_{N}$ are significantly lower than the other parameters $(p<0.05$ for all pairs of parameters with $\mathrm{EDT}_{N}$ and $T_{N}$ ) except between $\mathrm{EDT}_{N}$ and $T_{N}$
$[F(1,23)=1.21, \quad p=0.651]$, between $\mathrm{EDT}_{N}$ and $T_{20, \text { oct }}$ $[F(1,23)=1.85, p=0.148]$, and between $T_{N}$ and $T_{20, \text { oct }}$ $[F(1,23)=2.24, p=0.059]$. For $P_{\mathrm{REV}}$, its coefficients of variation are significantly higher than the other parameters $\left(p<0.01\right.$ for all pairs of parameters with $\left.P_{\mathrm{REV}}\right)$ both in experiments A.1 and A.2. On the basis of this result, Table II tabulates the psychoacoustic reverberance parameters that are significantly better matches to reverberance than each ISO parameter. Table II shows that $\mathrm{EDT}_{N}$ is a better match to reverberance in experiments A.1 and A.2, while $T_{N}$ is a better match only in experiment A.2.

For the analysis of the selected samples (for which the range of SPL across the samples is less than $1 \mathrm{~dB}$ ), in the two experiments all the tested parameters are similarly matched to the subject responses. Results of a two-sample $f$ test show that the differences in coefficients of variation between the parameters are not statistically significant ( $p>0.05$ for all pairs of the parameters). The interesting result here is that $P_{\mathrm{REV}}$ is not an outlier for these samples, which were very similar in SPL.

\section{B. Reverberance evaluation experiments}

\section{Experiments}

van Dorp Schuitman et al. (2013) conducted four experiments in which subjects listened to four sets of binaural audio samples and rated four acoustic qualities, namely, reverberance, clarity, apparent source width, and listener envelopment, on a range from "very low" to "very high." Only the responses for reverberance are used in the present study. Each set of binaural recordings represents different acoustic conditions as listed in Table III. For the samples, an anechoic solo cello recording and anechoic speech were convolved with the four sets of measured or simulated binaural room impulse responses (BRIRs).

The experiments were conducted with a double-blind task, following a so-called "mixed procedure" method proposed by Chevret and Parizet (2007), which is a mix between a paired comparison and a direct evaluation method. Using this method, the subjects are allowed to apply direct rating to the samples using a slider on the screen and then the collected subject responses can be sorted from the highest to lowest rating, allowing for paired comparisons by fine-tuning the ratings. Chevret and Parizet applied this method to assess the perceived quality of car door closing sounds and showed that this method yields the same quality of responses as a paired comparison test, but with much shorter testing times. The same test procedure has been followed in experiments $\mathrm{B}$, where subjects were asked to rate

TABLE II. Psychoacoustic reverberance parameters that are significantly better matches to reverberance than each ISO parameter in experiments A.1 and A. 2 for all samples $(p<0.05)$.

\begin{tabular}{lcccc}
\hline \hline & $\mathrm{EDT}_{\text {oct }}$ & $\mathrm{EDT}_{\text {mid }}$ & $T_{20, \text { oct }}$ & $T_{20, \text { mid }}$ \\
\hline A.1 & $\mathrm{EDT}_{N}$ & $\mathrm{EDT}_{N}$ & $\mathrm{EDT}_{N}$ & $\mathrm{EDT}_{N}$ \\
$\mathrm{A.2}$ & $\mathrm{EDT}_{N}, T_{N}$ & $\mathrm{EDT}_{N}, T_{N}$ & None & $\mathrm{EDT}_{N}, T_{N}$ \\
\hline \hline
\end{tabular}


TABLE III. An overview of the properties of experiments B.

\begin{tabular}{|c|c|c|c|c|c|}
\hline Experiment number & Number of rooms & Room type & Number of subjects & Loudness normalized & Usage \\
\hline B.1 & 9 & Virtual (realistic) & 5 & Yes & Validation \\
\hline B. 2 & 8 & Virtual (unrealistic) & 5 & Yes & Validation \\
\hline B. 3 & 10 & Real & 15 & No & Training \\
\hline B. 4 & 10 & Real & 15 & Yes & Validation \\
\hline
\end{tabular}

room acoustical qualities like "reverberance" using this mixed procedure.

As seen in Table III, experiments B.1 and B.2 included "virtual" rooms, for which the BRIRs were simulated using an acoustic shoebox model (van Dorp Schuitman, 2011). Five expert subjects participated in these experiments. They were from the acoustics department at TU Delft with indepth knowledge about the room acoustical parameters and had experience in assessing those parameters. All subjects reported normal hearing. Before the start of the experiments, the subjects received instructions (including audio examples) explaining reverberance.

The main difference between the two tests is that for experiment B.1 "realistic" rooms were chosen with a large spread in RT, whereas rooms for experiment B.2 have more "non-realistic" properties in terms of dimensions, shape, and spatial distribution of absorption (e.g., one of the tested rooms had a long RT but side walls that were fully absorbing). In both tests, the samples were normalized to the same estimated loudness using the Replaygain 1.0 algorithm (Robinson, 2001).

For experiments B.3 and B.4, the BRIRs were measured using the ITA dummy head (Schmitz, 1995) for convolutions with the anechoic samples, and RIRs were measured using an omnidirectional microphone (Type 4134, Brüel and Kjær, Nærum) for the calculation of ISO parameters. The only difference between the two experiments is that for experiment B.4 all samples were normalized, whereas for experiment B.3 the samples retained their original loudness differences. Tables IV-VII list $T_{20 \text {,mid }}$ EDT mid $_{\text {and }} L_{\text {Aeq }}$ (taking the transfer function of the headphones into account) of the samples used in experiments B.1-B.4. Fifteen subjects participated in experiments B.3 and B.4. They consisted mostly of students with mixed musical experiences and preferences, and received the same instructions (including the audio examples) explaining reverberance as in experiments B.1 and B.2. None of the subjects reported hearing problems.

\section{Results}

The performances of the parameters in experiments B are compared by calculating correlation coefficients between

TABLE IV. $\mathrm{T}_{20 \text {,mid }}$, EDT $\mathrm{mid}_{\text {mid }}$, and $\mathrm{L}_{\text {Aeq }}$ values of the samples used in experiment B.1.

\begin{tabular}{lccccccccc}
\hline \hline Room number & 1 & 2 & 3 & 4 & 5 & 6 & 7 & 8 & 9 \\
\hline$T_{20, \text { mid }}(\mathrm{s})$ & 0.01 & 0.33 & 0.72 & 0.73 & 1.81 & 1.91 & 1.40 & 2.02 & 6.92 \\
EDT $_{\text {mid }}(\mathrm{s})$ & 0.01 & 0.08 & 0.85 & 0.83 & 2.05 & 1.73 & 1.39 & 2.14 & 7.01 \\
$L_{\text {Aeq }}$, cello $(\mathrm{dB})$ & 68.9 & 68.3 & 68.7 & 69.0 & 68.7 & 68.9 & 69.2 & 69.7 & 68.5 \\
$L_{\text {Aeq }}$, speech $(\mathrm{dB})$ & 64.7 & 65.2 & 65.1 & 65.1 & 65.7 & 65.3 & 64.9 & 65.8 & 66.3 \\
\hline \hline
\end{tabular}

the parameters and the subject responses, as in the study of van Dorp Schuitman et al. (2013). The correlation coefficient indicates the strength and direction of the linear relationship between two factors, and its value ranges from -1 to +1 (Privitera, 2015). Therefore, an ideal reverberance parameter would yield a correlation coefficient of $r=1$. As each subject may have rated "very low" and "very high" differently on the continuous scale, the subject responses were normalized according to ITU-R BS.1284-1 (ITU-R, 2003) to compensate for variations in interpretation of the scale

$$
z_{i}=\frac{x_{i}-\bar{x}_{i}}{\sigma_{i}} \sigma+\bar{x},
$$

where $z_{i}$ is the normalized results for subject $i, x_{i}$ are the results for subject $i, \bar{x}_{i}$ is the mean result for this subject, and $\sigma_{i}$ is the standard deviation. $\bar{x}$ and $\sigma$ are the mean and the standard deviation, respectively, for all subjects.

Tables VIII and IX tabulate values of the correlation coefficient between the normalized subject responses (hereafter, subject responses) and the reverberance parameters for the cello samples and the speech samples, respectively. All the $r$-values in Tables VIII and IX are statistically significant $(p<0.05)$, except those showing negative correlations in Table IX. The highest correlation observed in each experiment is in boldface.

In Tables VIII and IX, $P_{\mathrm{REV}}$ is most highly correlated with the subject responses in experiments B.1, B.2 (only for the speech samples), and B.4. The significance of the difference between correlation coefficients is assessed with a Meng's z-test (Meng et al., 1991), which is a z-test on the basis of the number of samples and the three sets of correlation coefficients (e.g., $r_{\mathrm{xz}}, r_{\mathrm{yz}}$, and $r_{\mathrm{xy}}$, where $x$ and $y$ are parameter values and $z$ signifies the subject responses). The result shows that the differences in correlation coefficient between $P_{\mathrm{REV}}$ and the other parameters are statistically significant $(z>1.5, p<0.01$ for all the possible pairs of parameters with $\left.P_{\text {REV }}\right)$. By contrast, the highest correlation coefficient of $\mathrm{EDT}_{N}$ in experiments B.3 is only statistically significant when it compares with $P_{\mathrm{REV}}$ for the cello samples $(n=10, z=1.87, p=0.031)$.

TABLE V. $\mathrm{T}_{20 \text {,mid }}$, $\mathrm{EDT}_{\text {mid }}$, and $\mathrm{L}_{\text {Aeq }}$ values of the samples used in experiment B.2.

\begin{tabular}{lcccccccc}
\hline \hline Room number & 1 & 2 & 3 & 4 & 5 & 6 & 7 & 8 \\
\hline$T_{20, \text { mid }}(\mathrm{s})$ & 1.27 & 1.21 & 1.25 & 1.75 & 1.77 & 1.82 & 1.72 & 1.98 \\
EDT $_{\text {mid }}(\mathrm{s})$ & 1.42 & 1.39 & 1.49 & 2.06 & 1.83 & 1.72 & 1.87 & 1.86 \\
$L_{\text {Aeq, }}$ cello (dB) & 69.5 & 69.3 & 69.2 & 69.9 & 69.3 & 68.8 & 69.2 & 69.1 \\
$L_{\text {Aeq }}$, speech (dB) & 66.2 & 65.6 & 66.2 & 65.7 & 66.1 & 65.2 & 66.2 & 66.1 \\
\hline \hline
\end{tabular}


TABLE VI. $\mathrm{T}_{20 \text {,mid }}$, $\mathrm{EDT}_{\text {mid }}$, and $\mathrm{L}_{\text {Aeq }}$ values of the samples used in experiment B.3.

\begin{tabular}{lcccccccccc}
\hline \hline Room number & 1 & 2 & 3 & 4 & 5 & 6 & 7 & 8 & 9 & 10 \\
\hline$T_{20, \text { mid }}(\mathrm{s})$ & 0.02 & 0.21 & 0.76 & 1.02 & 1.21 & 1.67 & 2.29 & 3.94 & 4.81 & 10.12 \\
EDT $_{\text {mid }}(\mathrm{s})$ & 0.02 & 0.13 & 0.79 & 0.98 & 0.88 & 1.44 & 1.12 & 4.83 & 2.11 & 9.84 \\
$L_{\text {Aeq }}$, cello $(\mathrm{dB})$ & 45.9 & 60.5 & 60.0 & 62.9 & 47.8 & 52.5 & 48.3 & 60.4 & 47.2 & 72.2 \\
$L_{\text {Aeq }}$, speech $(\mathrm{dB})$ & 42.8 & 59.4 & 58.5 & 61.2 & 45.8 & 48.4 & 46.0 & 58.8 & 44.2 & 71.2 \\
\hline \hline
\end{tabular}

Based on the results of a Meng's z-test, Table X tabulates psychoacoustic reverberance parameters that are significantly more highly correlated with reverberance than each ISO parameter. As shown in Table $\mathrm{X}, P_{\mathrm{REV}}$ is a significantly better match to reverberance than all the ISO parameters in experiments B.1, B.2 (only for the speech samples), and B.4. $\mathrm{EDT}_{N}$ is significantly better correlated to reverberance than only some ISO parameters in experiments B.1 and B.4, but $T_{N}$ is not a significantly better match to reverberance than any ISO parameter in experiments B.

With respect to the effect of sample type (cello/speech), results of a paired-sample $t$-test show that the sample type does not significantly affect the tested parameters and the subject responses, except $P_{\mathrm{REV}}$ in experiment B.1 $[t(16)=-2.39$, $p=0.030]$ and in experiment B.2 $[t(14)=-13.94, p<0.01]$.

\section{DISCUSSION}

This study examines the performance of psychoacoustic reverberance parameters in various listening conditions, for reverberance-matched samples (experiments A), and for samples from which subjective scale values have been derived (experiments B). In the first case, ideally there should be no variation in reverberance between the reverberance-matched samples, and so a small coefficient of variation is sought. In the second case, performance is indicated by correlation between the reverberance parameter and the subjective scale values. A two-factor $f$-test and a Meng's $z$-test were executed to investigate the significance of difference between coefficients of variation in experiments $\mathrm{A}$, and between correlation coefficients in experiments $\mathrm{B}$, respectively.

The two parameters $\mathrm{EDT}_{N}$ and $T_{N}$ were proposed because in the experiments by Lee and Cabrera (2010) and Lee et al. (2012a) they both seemed to be plausible approaches, and it was not clear which was better as a reverberance parameter. Since then, Lachenmayr (2016) compared these in a subjective test using a room with electro-acoustically augmented reverberation, finding that $\mathrm{EDT}_{N}$ provided better performance than $T_{N}$ (and also better than other parameters tested). Results of the current study (especially from experiments B) provide

TABLE VII. $\mathrm{T}_{20 \text {,mid }}, \mathrm{EDT}_{\text {mid }}$, and $\mathrm{L}_{\text {Aeq }}$ values of the samples used in experiment B.4.

\begin{tabular}{lcccccccccc}
\hline \hline Room number & 1 & 2 & 3 & 4 & 5 & 6 & 7 & 8 & 9 & 10 \\
\hline$T_{20, \text { mid }}(\mathrm{s})$ & 0.02 & 0.21 & 0.76 & 1.02 & 1.21 & 1.67 & 2.29 & 3.94 & 4.81 & 10.12 \\
EDT $_{\text {mid }}(\mathrm{s})$ & 0.02 & 0.13 & 0.79 & 0.98 & 0.88 & 1.44 & 1.12 & 4.83 & 2.11 & 9.84 \\
$L_{\text {Aeq }}$, cello $(\mathrm{dB})$ & 69.3 & 67.1 & 68.8 & 68.1 & 69.5 & 66.0 & 64.2 & 65.2 & 64.5 & 65.7 \\
$L_{\text {Aeq }}$, speech (dB) & 70.1 & 69.3 & 69.7 & 69.7 & 70.2 & 65.9 & 65.8 & 66.3 & 64.9 & 67.7 \\
\hline \hline
\end{tabular}

TABLE VIII. The correlation coefficients between the tested reverberance parameters and the normalized subject responses collected from experiments B with cello samples. The highest correlation observed in each experiment is in boldface.

\begin{tabular}{lccccccc}
\hline \hline & EDT $_{\text {oct }}$ & EDT $_{\text {mid }}$ & $T_{20, \text { oct }}$ & $T_{20, \text { mid }}$ & EDT $_{N}$ & $T_{N}$ & $P_{\text {REV }}$ \\
\hline B.1 & 0.88 & 0.85 & 0.85 & 0.84 & 0.89 & 0.85 & $\mathbf{0 . 9 9}$ \\
B.2 & 0.84 & 0.83 & $\mathbf{0 . 9 7}$ & 0.96 & 0.32 & 0.93 & 0.87 \\
B.3 & 0.88 & 0.88 & 0.86 & 0.86 & $\mathbf{0 . 9 3}$ & 0.84 & 0.77 \\
B.4 & 0.78 & 0.78 & 0.78 & 0.79 & 0.87 & 0.81 & $\mathbf{0 . 9 5}$ \\
\hline \hline
\end{tabular}

further support for the use of $\mathrm{EDT}_{N}$ (rather than $T_{N}$ ) as a reverberance parameter.

For $P_{\mathrm{REV}}$, the best performance is observed in experiments B.1 and B.4, which tested a small range of SPL (less than $5 \mathrm{~dB}$ due to the SPL adjustment for constant loudness) and large range of $T_{20}$ (more than $5.0 \mathrm{~s}$ ). Considering that the samples in experiment B.3 are the same as those in experiment B.4, apart from their SPLs, $P_{\mathrm{REV}}$ is evidently overly sensitive to variation in SPL. This high sensitivity to the SPL is, in part, because the non-linear adaptation loops in the auditory model yield overshoots that are sometimes too large. The high sensitivity to the SPL can be seen in Fig. 2, and is also the main reason why $P_{\mathrm{REV}}$ yields lower values of the coefficient of variation in experiments A for the selected samples (for which the SPL range is less than $1 \mathrm{~dB}$ ) than for all the samples (in which SPL range exceeded 20). Hence, $P_{\mathrm{REV}}$ appears to be most useful for loudness-matched samples, or at least for samples across which the SPL varies little. It should be noted that the ISO parameters also yield lower values of the coefficient of variation for the selected samples in experiments A. However, unlike $P_{\mathrm{REV}}$, this is because they do not consider the SPL influence on reverberance, and therefore the elimination of this influence by analysing the selected samples results in values closer matching reverberance.

$P_{\mathrm{REV}}$ also appears to be particularly useful for evaluating reverberance of speech, and it significantly better correlates with the subjective responses than any of the conventional parameters in all of the speech experiments, except in experiment B.3 (see Table X). In Table IX, EDT ${ }_{N}$ $(r=0.84)$ achieves a higher correlation coefficient than $P_{\mathrm{REV}}(r=0.81)$, but those correlations are not significantly different according to a Meng's $z$-test $(n=10, z=0.41$, $p=0.341)$.

The most remarkable performance from $P_{\mathrm{REV}}$ is in experiment B.2, where, for speech samples, it achieves a

TABLE IX. The correlation coefficients between the tested reverberance parameters and the normalized subject responses collected from experiments $\mathrm{B}$ with speech samples. All the $r$-values in the table are significant at a confidence level of $95 \%(p<0.05)$, except the underscored values. The highest correlation observed in each experiment is in boldface.

\begin{tabular}{cccccccc}
\hline \hline & EDT $_{\text {oct }}$ & EDT $_{\text {mid }}$ & $T_{20, \text { oct }}$ & $T_{20, \text { mid }}$ & EDT $_{N}$ & $T_{N}$ & $P_{\text {REV }}$ \\
\hline B.1 & 0.83 & 0.80 & 0.79 & 0.79 & 0.85 & 0.79 & $\mathbf{0 . 9 6}$ \\
B.2 & $-\underline{-0.60}$ & $-\frac{0.55}{\underline{7}}$ & $-\underline{0.26}$ & $-\underline{-0.30}$ & $-\underline{-0.31}$ & $-\underline{-0.29}$ & $\mathbf{0 . 8 8}$ \\
B.3 & 0.76 & 0.76 & 0.74 & 0.74 & $\mathbf{0 . 8 4}$ & 0.70 & 0.81 \\
B.4 & 0.73 & 0.73 & 0.72 & 0.73 & 0.85 & 0.75 & $\mathbf{0 . 9 6}$ \\
\hline \hline
\end{tabular}


TABLE X. Psychoacoustic reverberance parameters that are significantly more highly correlated with reverberance in experiments $\mathrm{B}(p<0.05)$.

\begin{tabular}{lcccc}
\hline \hline & EDT $_{\text {oct }}$ & $\mathrm{EDT}_{\text {mid }}$ & $T_{20, \text { oct }}$ & $T_{20, \text { mid }}$ \\
\hline B.1 cello & $P_{\mathrm{REV}}$ & $\mathrm{EDT}_{N}, P_{\mathrm{REV}}$ & $\mathrm{EDT}_{N}, P_{\mathrm{REV}}$ & $\mathrm{EDT}_{N}, P_{\mathrm{REV}}$ \\
B.1 speech & $P_{\mathrm{REV}}$ & $\mathrm{EDT}_{N}, P_{\mathrm{REV}}$ & $\mathrm{EDT}_{N}, P_{\mathrm{REV}}$ & $\mathrm{EDT}_{N}, P_{\mathrm{REV}}$ \\
B.2 cello & - & - & - & - \\
B.2 speech & $P_{\mathrm{REV}}$ & $P_{\mathrm{REV}}$ & $P_{\mathrm{REV}}$ & $P_{\mathrm{REV}}$ \\
B.3 cello & - & - & - & - \\
B.3 speech & - & - & - & - \\
B.4 cello & $P_{\mathrm{REV}}$ & $P_{\mathrm{REV}}$ & $P_{\mathrm{REV}}$ & $P_{\mathrm{REV}}$ \\
B.4 speech & $\mathrm{EDT}_{N}, P_{\mathrm{REV}}$ & $\mathrm{EDT}_{N}, P_{\mathrm{REV}}$ & $P_{\mathrm{REV}}$ & $P_{\mathrm{REV}}$ \\
\hline \hline
\end{tabular}

high positive correlation while no other parameter yields a significant correlation. The comparative effectiveness of $P_{\mathrm{REV}}$ for speech can be explained by considering how it is calculated: The auditory model splits a running signal into a direct stream and a reverberant stream, but quasi-stationary ("legato") passages in the music samples provide less opportunity for the algorithm to the reverberant stream than speech samples.

Other issues arising from analysis of a running signal suggest that $P_{\mathrm{REV}}$ is more appropriate for estimating a relative change in reverberance, rather than absolute reverberance. For example, $P_{\mathrm{REV}}$ in experiments $\mathrm{B}$ changes by $15 \%$ on average when $1.0 \mathrm{~s}$ silence is added to the end of the samples, and by $7 \%$ and $14 \%$ on average when applying a $1.0 \mathrm{~dB}$ and $2.0 \mathrm{~dB}$ offset in calibration level, respectively. Note that adding $1.0 \mathrm{~s}$ silence changes the $L_{\mathrm{Aeq}}$ of the samples, but for this analysis the initial $L_{\text {Aeq }}$ values (as in Tables IV-VII) are used in order to explore the influence of this change on $P_{\mathrm{REV}}$ when a desired playback level is determined. Recently, the sensitivity of $P_{\mathrm{REV}}$ to the SPL was investigated further by Osses Vecchi et al. (2017), who showed that the effect of SPL on $P_{\mathrm{REV}}$ is dependent on the input spectrum. They suggested further research to investigate the effect of spectral presentation level on reverberance. The outcomes could be used to further improve the model and the robustness of $P_{\mathrm{REV}}$.

However, as long as the same change (e.g., the same calibration offset) is applied to all the samples consistently, the correlation coefficient between $P_{\mathrm{REV}}$ and the subjective responses changes by less than 0.02 . Furthermore, the fact that $P_{\mathrm{REV}}$ is a unitless parameter (so its value is not intuitively interpreted) also suggests the use of $P_{\mathrm{REV}}$ for the estimation of a relative change in reverberance. In contrast, $T_{N}$ and $\mathrm{EDT}_{N}$ are robust to calibration error, and use an intuitive and familiar unit of time in seconds (like $T_{20}$ and EDT). Thus, they appear to be also relevant for estimation of the absolute reverberance.

In Sec. II, the behaviours of $T_{N}, \mathrm{EDT}_{N}$, and $P_{\mathrm{REV}}$ are compared over a very wide range of SPLs. At very high SPLs, it is reasonable to expect reverberance not to increase with the SPL, in part, because the middle ear reflex provides a $12-14 \mathrm{~dB}$ reduction for an intense sound (Howard and Angus, 2013). While the middle ear reflex has a frequency limit and latency (Howard and Angus, 2013), music and speech are sustained enough and have a wide frequency spectrum for this auditory characteristic to be effective. Based on this assumption, $T_{N}$ and $\mathrm{EDT}_{N}$ appear to reasonably account for the limited effect of SPL at very high levels. This result is likely attributable to functions in the TVL for the SPL dependency of spectral masking. It should be noted that the TVL and the auditory model used for the calculation of $P_{\mathrm{REV}}$ do not include any function for the middle ear reflex.

Overall, the results of this study suggest that further improvements can be made to psychoacoustic reverberance parameters. The calculations of $P_{\mathrm{REV}}$ and $\mathrm{EDT}_{N}\left(\right.$ or $T_{N}$ ) are both based on psychoacoustic modeling, but follow quite different approaches. Hence, the prospects for an improved model that emulates psychoacoustic processes more closely appear to be promising. In future work, it would be interesting to examine how $\mathrm{EDT}_{N}$ and $T_{N}$ could be extended to model the spatial influences on reverberance using binaural loudness modeling, perhaps augmented by interaural cross correlation. This approach can also be applied to $P_{\mathrm{REV}}$ as it processes the two channel outputs of the auditory model (i.e., for left and right ears) separately. Another interesting future work would be to test $T_{N}, \mathrm{EDT}_{N}$, and $P_{\mathrm{REV}}$ for reverberance of a non-single-exponential sound decay. As shown by Jeong and Joo (2017), the ISO parameters are not accurate in such a condition. The incorporation of human factors in the reverberance estimation, like in $T_{N}, \mathrm{EDT}_{N}$, and $P_{\mathrm{REV}}$, should benefit the reverberance estimation for a multiexponential sound decay.

\section{CONCLUSION}

The respective performances of $T_{N}, \mathrm{EDT}_{N}$, and $P_{\mathrm{REV}}$ are compared to each other, as well as to $T_{20}$ and EDT. Results of this study suggest the use of $\mathrm{EDT}_{N}$ for music samples and not for speech (whereas $P_{\mathrm{REV}}$ is effective for speech samples). $T_{N}$ and $\mathrm{EDT}_{N}$ have not been previously tested with speech, and it appears that further development would be needed for them to predict reverberance of speech well. As $P_{\mathrm{REV}}$ is derived from a running signal, it is affected by the sample type and the processing of a sample. $P_{\mathrm{REV}}$ appears to be excessively sensitive to the SPL. For this reason, $P_{\mathrm{REV}}$ is more appropriate for the estimation of a relative change in reverberance for samples of similar SPL, while the RIRbased parameters, $T_{N}$ and $\mathrm{EDT}_{N}$, are also good for the estimation of the absolute reverberance. $\mathrm{EDT}_{N}$ is favoured over $T_{N}$ as a predictor of reverberance. The results of this study identifying the strengths and weaknesses of $T_{N}, \mathrm{EDT}_{N}$, and $P_{\mathrm{REV}}$ provide a basis for future research into improving these parameters, and provide a general guideline to users of these parameters.

\section{ACKNOWLEDGMENTS}

The authors thank the experiment participants and the members of the Advanced Acoustic Information Systems Laboratory at the Research Institute of Electrical Communication at Tohoku University in Japan. The visit to Tohoku University was supported by a University of Sydney Internationalization Program Development Fund grant, and a grant by the Global $\mathrm{CoE}$ program CERIES, Tohoku 
University. Experiments $B$ in this paper and the development of the auditory model for calculating $P_{\mathrm{REV}}$ was supported by grants from the Dutch technology foundation STW, Project No. DTF.7459.

Barron, M. (1988). "Subjective study of British symphony concert halls," Acustica 66, 1-14.

Breebaart, D. J. (2001). "Modelling binaural signal detection," Ph.D. thesis, Eindhoven University of Technology, Eindhoven, The Netherlands.

Breebaart, D. J., van de Par, S., and Kohlrausch, A. (2001). "Binaural processing model based on contralateral inhibition. I. Model structure," J. Acoust. Soc. Am. 110, 1074-1088.

Cabrera, D., Lee, D., Yadav, M., and Martens, W. L. (2011). "Decay envelope manipulation of room impulse responses: Techniques for auralization and sonification," in Proceedings of Acoustics 2011, Gold Coast, Australia.

Chalupper, J., and Fastl, H. (2002). "Dynamic loudness model (DLM) for normal and hearing-impaired listeners," Acta. Acust. Acust. 88, 378-386.

Chevret, P., and Parizet, E. (2007). "An efficient alternative to the paired comparison method for the subjective evaluation of a large set of sounds," in Proceedings of the 19th International Congress on Acoustics, Madrid, Spain.

Denon Professional Test CDs. (1988). CD-ROM, Nippon Columbia, Japan.

Farina, A., and Ayalon, R. (2003). "Recording concert hall acoustics for posterity," in Proceedings of the 24th AES Conference, Banff, Canada.

Fastl, H., and Zwicker, E. (2007). Psychoacoustics: Facts and Models, 3rd ed. (Springer, Berlin), Chaps. 3, 4, 6, and 8 .

Glasberg, B. R., and Moore, B. C. J. (2002). "A model of loudness applicable to time-varying sounds," J. Audio. Eng. Soc. 50, 331-342.

Griesinger, D. (1997). "The psychoacoustics of apparent source width, spaciousness and envelopment in performance spaces," Acta. Acust. Acust. 83, 721-731.

Hase, S., Takatsu, A., Sato, S., Sakai, H., and Ando, Y. (2000). "Reverberance of an existing hall in relation to both subsequent reverberation time and SPL," J. Sound. Vib. 232, 149-155.

Howard, D. M., and Angus, J. A. S. (2013). Acoustics and Psychoacoustics, 4th ed. (Focal, New York), Chap. 2.

ISO 3382-1. (2009). "Acoustics-Measurement of room acoustic parameters-Part 1: Performance spaces" (International Organization for Standardization, Geneva, Switzerland).

ITU-R BS. 1284-1. (2003). "General methods for the subjective assessment of sound quality" (International Telecommunication Union Radiocommunication Sector, Geneva Switzerland).

Jeong, D., and Joo, H. (2017). "Prediction of reverberance in rooms with simulated non-single-exponential sound decays," Appl. Acoust. 125, 136-146.
Lachenmayr, W. (2016). "Loudness-weighting of reverberation using electronic room enhancement," in Proc. 60th Audio Eng. Soc. Int. Conf., Leuven, Belgium.

Lee, D., and Cabrera, D. (2010). "Effect of listening level and background noise on the subjective decay rate of room impulse responses: Using timevarying loudness to model reverberance," Appl. Acoust. 71, 801-811.

Lee, D., Cabrera, D., and Martens, W. L. (2012a). "The effect of loudness on the reverberance of music: Reverberance prediction using loudness models," J. Acoust. Soc. Am. 131, 1194-1205.

Lee, D., Cabrera, D., and Martnes, W. L. (2012b). "Accounting for listening level in the prediction of reverberance using early decay time," Acoust. Aust. 40, 103-110

Lokki, T., Pätynen, J., Kuusinen, A., and Tervo, S. (2012). "Disentangling preference ratings of concert hall acoustics using subjective sensory profiles," J. Acoust. Soc. Am. 132, 3148-3161.

Mason, R., Brookes, T., and Rumsey, F. (2004). "Development of the interaural cross-correlation coefficient into a more complete auditory width prediction model," in Proceedings of the 18th International Congress on Acoustics, Kyoto, Japan.

Meng, X.-L., Rosenthal, R., and Rubin, D. B. (1991). "Comparing correlated correlation coefficients," Psychol. Bull. 111, 172-175.

Moore, B. C. J., and Glasberg, B. R. (2007). "Modeling binaural loudness," J. Acoust. Soc. Am. 121, 1604-1612.

Morimoto, M., and Asaoka, A. (2004). "Multi-dimensional analysis of 'Reverberance'," in Proceedings of the 18th International Congress on Acoustics, Kyoto, Japan.

Osses Vecchi, A., Kohlrausch, A., Lachenmayr, W., and Mommertz, E. (2017). "Predicting the perceived reverberation in different room acoustic environments using a binaural auditory model," J. Acoust. Soc. Am. 141, EL381-EL387.

Privitera, G. J. (2015). Statistics for the Behavioural Sciences, 2nd ed. (Sage, Los Angeles, CA), Chap. 15.

Robinson, D. (2001). Replay gain-A proposed standard, available at http:// wiki.hydrogenaud.io/index.php?title=ReplayGain_specification (Last viewed 7/7/2017).

Rumsey, F. (2002). "Spatial quality evaluation for reproduced sound: Terminology, meaning, and a scene-based paradigm," J. Audio. Eng. Soc. 50, 651-666

Schmitz, A. (1995). "Ein neues digitales Kunstkopfmeßsystem" ("A new digital artificial head measuring system"), Acustica 81, 416-420.

Teret, E., Pastore, M. T., and Braasch, J. (2017). "The influence of signal type on perceived reverberance," J. Acoust. Soc. Am. 141, 1675-1682.

van Dorp Schuitman, J. (2011). "Auditory modelling for assessing room acoustics," Ph.D. thesis, Delft University of Technology, Delft, Netherlands.

van Dorp Schuitman, J., de Vries, D., and Lindau, A. (2013). "Deriving content-specific measures of room acoustic perception using a binaural, nonlinear auditory model," J. Acoust. Soc. Am. 133, 1572-1585. 